\title{
Philosophiques
}

\section{Pensée, langage et perception}

\section{Denis Fisette}

Volume 18, numéro 2, automne 1991

URI : https://id.erudit.org/iderudit/027153ar

DOI : https://doi.org/10.7202/027153ar

Aller au sommaire du numéro

Éditeur(s)

Société de philosophie du Québec

ISSN

0316-2923 (imprimé)

1492-1391 (numérique)

Découvrir la revue

Citer cet article

Fisette, D. (1991). Pensée, langage et perception. Philosophiques, 18(2), 79-100. https://doi.org/10.7202/027153ar

\section{Résumé de l'article}

La parution récente d'un livre de M. Dummett sur les origines de la philosophie analytique sert ici de prétexte pour examiner à nouveau la question de la relation entre la théorie frégéenne de la signification et la théorie husserlienne de l'intentionnalité. Dummet défend la thèse suivant laquelle les pensées et le sens ont un caractère essentiellement linguistique et qu'ils sont incompatibles avec la conception husserlienne du contenu intentionnel. Nous examinerons les arguments qu'il fait valoir pour l'autonomie du langage et de la signification relativement à la pensée conceptuelle et la légitimité de la distinction entre pensée au sens propre et " pensée primitive » qui est introduite afin de rendre compte du cas de la perception.
Ce document est protégé par la loi sur le droit d'auteur. L'utilisation des services d'Érudit (y compris la reproduction) est assujettie à sa politique d'utilisation que vous pouvez consulter en ligne.

https://apropos.erudit.org/fr/usagers/politique-dutilisation/ 


\title{
PENSÉE, LANGAGE ET PERCEPTION*
}

\author{
par Denis Fisette
}

RÉSUMÉ. La parution récente d'un livre de M. Dummett sur les origines de la philosophie analytique sert ici de prétexte pour examiner à nouveau la question de la relation entre la théorie frégéenne de la signification et la théorie husserlienne de l'intentionnalité. Dummet défend la thèse suivant laquelle les pensées et le sens ont un caractère essentiellement linguistique et qu'ils sont incompatibles avec la conception husserlienne du contenu intentionnel. Nous examinerons les arguments qu'il fait valoir pour l'autonomie du langage et de la signification relativement à la pensée conceptuelle et la légitimité de la distinction entre pensée au sens propre et " pensée primitive " qui est introduite afin de rendre compte du cas de la perception.

ABSTRACT. In a recent book, Ursprünge der analytischen Philosophie, M. Dummett takes a new look at the relationship between Frege's theory of meaning and Husserl's doctrine of intentionality. He argues for the autonomy of linguistic meaning relative to conceptual thought and his arguments are supposed to show that Frege's "Sinne " are incompatible with Husserl's intentional content. I argue that once Dummett's understanding of the intentional content as something psychological is discarded, Husserl's phenomenology can be seen as an extension of, and perhaps a complement to, what Dummett calls Frege's theory of meaning.

* Cette étude reprend la question du rapport entre Husserl et Frege là où je l'avais laissée dans mon article "Husserl et Frege: les remarques de $M$. Durnmett sur la généralisation de la signification " destiné à un collectif portant sur l'oeuvre de M. Dummett. Je tiens à remercier P. Dumouchel, D. Laurier, R. Nadeau, S. Robert, M. Seymour soit pour leurs encouragements ou leurs commentaires. La présente étude a été rendue possible grâce à une subvention du CRSHC. 
Man könnte [Husserl und Frege] mit dem Rhein und der Donau vergleichen, die nicht weit voneinander entspringen und später ein stück weit nahezu parallel fließsen, dann aber in völlig veschiedene Richtungen strömen und in verschiedene Meere münden. Wie ist es dazu gekommen?

M. Dummett

Ursprünge der analytischen Philosophie est la traduction allemande d'un manuscrit de langue anglaise qui a servi de base à une série de conférences que $M$. Dummett a présentées au printemps de 1987 à l'université de Bologne ${ }^{1}$. Contrairement à ce que suggère le titre, "Origines de la philosophie analytique » n'est pas une étude historique du type de celle que l'on serait en droit d'attendre d'un historien de la philosophie. Dummett $s^{\prime}$ emploie plutôt à expliciter les thèses qu'il a mises de l'avant dans ses ouvrages désormais classiques sur G. Frege ${ }^{2}$. Rompant avec une tradition consacrée qui voyait dans G.E. Moore et B. Russell l'origine de la philosophie analytique, Dummett soutient que cette paternité revient à Frege dont le mérite est d'avoir fixé l'objet et la méthode de la philosophie analytique. L'objet principal de la philosophie devient l'analyse de la structure de la pensée (Gedanke) et la seule méthode y donnant accès serait l'analyse du langage. C'est en partant de cette caractérisation, qui ferait de Frege le premier philosophe du langage, que Dummett cherche à singulariser la place qui lui revient dans l'histoire de la philosophie. Frege aurait provoqué un changement de perspective en philosophie en assignant à sa théorie de la signification (Sinn) le rôle que jouait traditionnellement l'épistémologie depuis Descartes pour la philosophie moderne. $C^{\prime}$ est en partie ce qui explique que l'une des préoccupations principales de ce livre porte sur la relation entre la phénoménologie de Husserl, plus particulièrement sa théorie de l'intentionnalité, et la théorie frégéenne de la signification. À cet égard, ce livre est intéressant ne serait-ce que parce que Dummett examine cette relation d'un point de vue différent de celui auquel nous a habitué depuis quelque temps la phénoménologie contemporaine.

Plus préoccupé par la légitimité de sa propre entreprise que par les jugements qu'il porte sur ses contemporains comme G. Evans, Dummett cherche à retracer sa filiation jusqu'aux origines de la philosophie analytique. Cette filiation prend évidemment la forme d'un arbre généalogique: le grand-père 
est B. Bolzano, Frege est le père et Dummett le descendant le plus direct, le fils légitime. On retrouve également B. Russell et G. E. Moore, mais ceux-ci ne représentent tout au plus que ses "grands-oncles" (p. 167). Husserl, à qui l'on doit la (re-)découverte de Bolzano, représente l'autre branche de cette génération, la lignée phénoménologique. Comme le remarque Dummett [1986: 37] en faisant abstraction de la distance qui sépare la philosophie analytique de la phénoménologie qui se pratique aujourd'hui sur le continent, un observateur de 1903 connaissant l'oeuvre de Husserl comme celle de Frege aurait peut-être remarqué quelques différences quant à leurs intérêts thématiques, mais il n'aurait certainement pas vu là deux orientations radicalement opposées. C'est pourquoi Dummett écrit:

On pourrait les comparer au Rhin et au Danube qui prennent source non loin l'un de l'autre pour ensuite suivre un cours presque parallèle pour un moment mais qui affluent ensuite dans des directions totalement différentes et qui se jettent dans des mers différentes. [1986: 37]

Dummett suggère que le parallèle auquel il fait allusion dans ce passage s'établit à l'époque des Recherches Logiques (1900/1) dont le thème principal est le concept de signification. La divergence, il la voit dans la phénoménologie des Idées Directrices (1913), divergence qui, selon lui, ira toujours croissante au fur et à mesure que Husserl progressera dans cette voie.

À partir des Idées Directrices, le thème central de la phénoménologie est l'intentionnalité. Comme l'indique Husserl au premier volume de cet ouvrage,

Le titre du problème qui embrasse toute la phénoménologie est l'intentionnalité. Il exprime en effet la propriété fondamentale de la conscience, et tous les problèmes phénoménologiques [...] s'y incorporent. [1950: 490]

Le concept central de la théorie husserlienne de l'intentionnalité est le "noème". Le "noème" que nous analyserons plus loin est le résultat de la combinaison de l'idée d'intentionnalité introduite par $\mathrm{F}$. Brentano (la directionnalité des actes mentaux) et d'une distinction équivalente à celle que Frege a fait valoir entre "Sinn" et "Bedeutung » dans sa théorie de la signification. Le noème est en fait l'élargissement de l'idée de signification à l'ensemble de toute l'activité d'un agent et c'est sur cette généralisation que repose toute l'entreprise de la phénoménologie. C'est précisément à ce titre que Dummett s'intéresse au concept 
de «noème» et plus particulièrement aux conséquences que cet élargissement entraînerait pour la théorie frégéenne de la signification. La première conséquence concerne l'objectivité de la composante essentielle de cette théorie, à savoir le sens (Sinn). Dummett craint qu'en généralisant ainsi la signification à l'ensemble des actes, Husserl ne la réduise à quelque chose comme une représentation ou une idée subjective et qu'il ne soit conduit, malgré lui, à une forme plus ou moins obscure d'idéalisme psychologisant. Cette critique, Dummett [1973, 1981] l'a adressée à quelques reprises à l'endroit de la phénoménologie. Ce qu'il y a de nouveau dans ces conférences, c'est qu'elle prend une forme plus systématique et qu'elle permet de mieux saisir les enjeux qui sont à l'origine des appréhensions de Dummett à l'égard de l'entreprise de Husserl mais aussi de celle de G. Evans qui fait aussi appel à un concept d'intentionnalité afin d'expliquer celui de signification.

II

Le livre se divise en 14 chapitres et il est complété d'un entretien auquel Dummett s'est prêté avec son traducteur J. Schulte. Il nous prévient dès le premier chapitre qu'il ne s'agit pas ici d'une étude historique, au sens étroit du terme, et que, pour des raisons thématiques, il sera surtout question de philosophes de langue allemande. Au chapitre suivant, Dummett distingue trois caractéristiques qui font de Frege un philosophe du langage. La première porte sur la relation entre le sens ou la signification et son expression linguistique. À la question de savoir ce qui prête sens à une expression, Dummett [1986: 21] répond que c'est "par le fait que le locuteur la comprend en ce sens". Mais en quoi consiste la compréhension d'un locuteur? Elle consiste dans la saisie de la référence, et plus précisément dans la saisie adéquate de la valeur de vérité d'une proposition. Car c'est en comprenant comment la référence est déterminée que le locuteur saisit ce qu'est le sens. Deuxièmement, Frege considère que la valeur de vérité est attribuable en premier lieu à la pensée. Une proposition ne peut être dite vraie ou fausse que dans un sens dérivé. Finalement, nous n'avons pas accès directement à la pensée, c'est-à-dire que la connaissance de la pensée passe par la saisie de la structure de la proposition qui en est l'expression (p. 15).

Au chapitre 3 et 4 sont introduites deux contributions importantes de Frege qui, selon Dummett, sont devenues des lieux communs en philosophie analytique. Il s'agit d'abord 
de l'indissociabilité des concepts de signification et de vérité (référence). La signification d'une expression est, comme nous l'avons dit, la manière par laquelle sa référence est donnée. Mais pour savoir quelle forme doit prendre une théorie de la signification, il faut une théorie de la référence (vérité). C'est pourquoi ces deux concepts sont indissociables.

La seconde contribution de Frege à la philosophie consiste dans l'expulsion des "Gedanken " (ou de ce qu'on appellerait volontiers aujourd'hui un "contenu de pensée », le "contenu d'un acte » ou encore le " contenu d'une attitude propositionnelle ") hors de la conscience. Le chapitre 4 soulève toute la question de l'anti-psychologisme que Frege partage avec Bolzano, Meinong mais aussi avec Husserl qui, dans Philosophie de l'Arithmétique, s'était commis à une forme de psychologisme mais qui, à partir des Recherches Logiques et sans doute sous l'influence de Frege [1970], a reconnu la nécessité de bien distinguer le caractère objectif des contenus de pensée des représentations subjectives de la pensée. Je n'insiste pas sur cette question qui a fait l'objet de nombreux débats au cours des dernières années ${ }^{3}$. C'est d'ailleurs ce que fait Dummett qui se contente de rappeler l'insistance avec laquelle Frege distingue les "Vorstellungen », qui sont subjectives au sens où elles diffèrent d'un agent à l'autre et qui ne sont pas itérables (communicables), des "Gedanken » dont le statut et le rôle correspondent à ce que Bolzano nomme « Sätze an sich» et Husserl le «sens noématique ». Beaucoup plus importante dans le contexte actuel est la question concernant l'attitude de Frege et de Husserl à l'égard de la relation entre les pensées et la perception sensible. Cette question fait l'objet des chapitres 5-12.

\section{III}

Au chapitre intitulé «le legs de Brentano», Dummett examine le contexte dans lequel s'est développée la théorie husserlienne de l'intentionnalité. L'on doit à $F$. Brentano d'avoir réactivé le concept scolastique d'intentionnalité afin de distinguer les phénomènes psychiques des phénomènes physiques. Selon lui, ce qui caractérise les actes de conscience, c'est la propriété de ce type d'actes d'être dirigés vers quelque chose. Dummett [1986: 39] interprète ce quelque chose comme l'objet d'un acte, comme un objet externe appartenant au monde extérieur. Ainsi, lorsque, par exemple, je promets de marier quelqu'un, l'objet de ma promesse n'est pas une simple représentation mais la personne elle-même. Ce qu'il est important de retenir ici, c'est 
que l'intentionnalité d'un acte est, pour Brentano, fonction de l'objet que cet acte vise. Cependant, comme l'a remarqué Dummett et plusieurs autres, cette conception fait face à de sérieuses difficultés, car si l'intentionnalité $d^{\prime}$ 'un acte est fonction de l'objet qu'il vise, qu'en est-il des cas où il n'y a aucun objet réel correspondant à l'acte? Qu'en est-il des cas d'illusion pour prendre l'exemple d'un acte de perception? Ces actes portent pourtant la marque de l'intentionnalité puisqu'ils sont dirigés vers quelque chose. Doit-on dire que, dans ce cas, l'objet appartient à la conscience à titre d'objet intentionnel? Et si tel était le cas, comment distinguer un objet réel $\mathrm{d}^{\prime}$ un objet intentionnel (ou mental)?

Telles sont les questions auxquelles la théorie husserlienne prétend apporter une réponse. La stratégie de Husserl consiste à ne retenir de la théorie de Brentano que l'idée de directionnalité d'un acte et de faire appel à une distinction analogue à celle dont Frege s'est servi dans sa théorie de la signification entre "Sinn" et "Bedeutung". Ainsi, par analogie avec une expression linguistique qui peut avoir un sens sans que lui corresponde aucun existant, un acte peut avoir un contenu, un sens, auquel ne correspond aucun objet. Le "contenu intentionnel " d'un acte est ce qu'il appelle à partir des Idées Directrices le "noème" et sa fonction est équivalente au «Sinn » frégéen. Ainsi, contrairement à la doctrine de Brentano suivant laquelle l'intentionnalité d'un acte est fonction de l'objet, le noème désigne cette propriété de l'acte qui en fait un acte intentionnel et qui rend possible la référence à l'objet. Qu'il y ait un noème auquel ne correspond aucun objet comme c'est le cas d'une perception trompeuse ne pose pas plus de problème que dans le cas d'une expression linguistique. Nous verrons bientôt que dans un tel cas, la conscience se comporte comme si il y en avait un.

En admettant que la distinction frégéenne explique la solution que Husserl propose aux difficultés rencontrées par la première théorie de Brentano, il faut maintenant se demander si le noème, que Husserl introduit dans les Idées Directrices, est compatible avec la conception frégéenne du sens. Dummett met en doute la légitimité de ce rapprochement en faisant valoir le caractère essentiellement linguistique du sens.

Un passage tiré de Ideen III (p. 89) nous informe sur l'origine et le sens du noème:

I. noème n'est rien d'autre que la généralisation (Verallgemeinerung) de l'idée de signification à la sphère de tous les actes. 
Par " généralisation ", Husserl [1950: 418] précise qu'il s'agit de l'élargissement du sens des mots "Bedeutung " et "Bedeuten" qui, à l'origine, appartiennent au domaine linguistique, à tous les actes, c'est-à-dire à toute l'expérience et à toute l'activité humaine - que ces actes soient ou non liés aux actes expressifs et au langage. La première conséquence est de détacher le sens de son véhicule linguistique et de faire de la signification linguistique une forme parmi d'autres d'intentionnalité.

Qu'en est-il maintenant de l'idée de signification dont il est question dans ce passage? Comme le démontre clairement toute la $\S 16$ de Ideen III dont ce passage est extrait, il ne $\mathrm{s}^{\prime}$ agit pas du concept de signification avec lequel opéraient les Recherches Logiques. Il est vrai que Husserl disposait de la distinction entre signification et objet dans cet ouvrage. Cependant, la signification y était conçue comme une "essence d'acte". Or, le noème ou ce qu'il appelle aussi la «signification noématique » est radicalement différente de la doctrine de la signification des Recherches Logiques: le noème n'est pas une essence d'acte mais bien son corrélat objectif ${ }^{4}$. C'est pourquoi il est difficile de faire suite aux remarques de Dummett [1988: 45, 85] qui attribue à Husserl une conception de la signification qui s'apparente à celle du type Humpty-Dumpty suivant laquelle la signification linguistique s'épuise dans l'acte qui lui prête signification ${ }^{5}$.

En tenant compte de cela, on peut se demander quelle est la relation entre le noème et le langage? C'est précisément sur cette question que Dummett oriente le débat. Il s'agit de la thèse de l'exprimabilité du contenu intentionnel que Husserl énonce dans ses Idées Directrices:

Tout "visé [Gemeint] en tant que tel ", toute visée [Meinung] au sens noématique (en entendant par là le noyau noématique) est susceptible, quel que soit l'acte, de recevoir une expression au moyen de "significations". [1950: 419]

Le mot "sens" (Sinn) est une abréviation pour "sens noématique» qui représente la composante essentielle, le noyau central du noème. Il s'agit de la signification au sens élargi qu'il appelle ici le "sens noématique " alors qu'il continue d'employer "signification" (Bedeutung) dans son acception courante, c'està-dire comme signification logique ou expressive [1950: 419]. La signification est alors comprise comme sens exprimé et la thèse est que tout sens, quel que soit le type d'acte, peut être exprimé linguistiquement. Corrélativement, le langage est conçu comme: 
Un médium spécifique [...] dont le propre est par essence de refléter toute autre intentionnalité quant à sa forme et à son contenu... [Husserl, 1950: 420]

Selon Dummett [1988: 36-7], Frege admet également que le sens est irréductible à la signification linguistique. Cependant, alors que Frege considère que le sens doit pouvoir être exhaustivement exprimé linguistiquement, Husserl réserve ce privilège au sens noématique considérant par ailleurs que les autres couches du noème ne peuvent pas être exprimées (pp. 97-8). C'est pourquoi la conception husserlienne du noème serait, suivant l'interprétation de Dummett, incompatible avec le sens frégéen et qu'elle rendrait impraticable le «tournant vers le langage ». Par conséquent, afin d'arriver, par la généralisation, à la conception husserlienne du noème, une conception du sens est requise qui diffère essentiellement de celle de Frege. Pour s'en convaincre, il suffit d'examiner le problème de la perception.

\section{IV}

Si l'introduction du concept de noème apporte une solution aux problèmes auxquels fait face la théorie de Brentano, Dummett [1981: 138] soutient qu'elle le fait au prix d'une forme "intellectualisée " d'idéalisme. Les critères essentiels à ce type d'idéalisme sont au nombre de trois. D'abord l'objet n'est accessible que d'une manière particulière. Husserl et Frege considèrent en effet que les objets physiques sont "transcendants ", c'està-dire que notre connaissance de la référence est toujours partielle et qu'elle n'est jamais adéquate à ce qui est donné en elle. Cela veut dire tout d'abord que l'existence de l'objet n'est pas récessaire à l'expérience. Deuxièmement, ce mode de donation ou de présentation est un "sens". Encore ici, il ne fait aucun doute que cette caractéristique du sens comme "mode de présentation " et comme "mode de détermination » revient également au noème [Husserl, 1950: § 131]. Le problème concerne plutôt la troisième thèse suivant laquelle il n'est intrinsèque à aucun sens d'avoir une référence correspondante. Selon Dummett, la réunion de ces trois thèses est inhérente à cette forme d'idéalisme. Cependant, on peut y échapper en évitant la troisième thèse, c'est-à-dire en s'assurant, comme le fait Frege, qu'à tout sens correspond une référence (p. 58). C'est donc cette troisième thèse qu'il faut maintenant examiner. La question est de savoir: si l'on admet avec Husserl que l'intentionnalité d'un acte n'est pas fonction de l'objet, et donc qu'un acte 
peut avoir un contenu sans qu'il y ait d'objet donné, alors de quel droit présuppose-t-on qu'il y a des objets hors de la conscience? En d'autres mots, quelle est la ligne de démarcation entre "référer à un objet " et se contenter de simplement le penser? Pour répondre à ces questions, prenons le cas de la perception auquel Dummett accorde beaucoup d'importance dans ses conférences.

Commençons par circonscrire la position de Husserl sur cette question. Selon lui, la différence essentielle entre la perception et les autres actes, par exemple l'imagination, c'est que la perception implique nos expériences sensorielles. Ainsi, lorsque nos sens sont affectés par un objet physique, nous faisons l'expérience de ce que Husserl appelle à partir des Idées Directrices la «hylè». La hylè est cet élément étrangé à la conscience avec lequel doit concorder le noème perceptuel. Sa fonction consiste à diminuer le nombre de noèmes applicables à un objet donné. Cependant, comme le remarque Dummett [1988: 54], la conception de Husserl n'est pas à confondre avec la théorie des "sense data" car la hylè n'est pas l'objet premier de la conscience. Ce qui est perçu, ce ne sont pas les impressions sensibles dont on inférerait l'existence de l'objet, mais c'est l'objet lui-même.

Un autre aspect important que mentionne Dummett relativement à cette problématique, c'est l'origine causale de la hylè, c'està-dire les coordonnées spatio-temporelles de l'objet à l'origine des stimuli qui irritent la surface des sens. Selon lui,

Il serait possible de faire valoir que le noème, qui remplit un acte de perception, comprend toujours une référence, médiatisée par le concept de causalité, à un objet déterminé, et ce dans la mesure où cet objet présente l'origine des impressions sensorielles du sujet. [1988: 100]

Mais Dummett [1988: 100] s'empresse d'ajouter que ce n'est pas le cas et que, par conséquent, cette théorie de la perception n'est pas apte à expliquer "comment un objet déterminé devient celui vers lequel un acte spécifique de perception est orienté ». Cette dernière remarque est pour le moins déroutante puisqu'il semble reconnaître par ailleurs le bien-fondé de la solution que Husserl prétendait apporter à ce problème dans la $\mathrm{VI}^{\mathrm{e}}$ des Recherches Logiques ( $\$ 5$ ) où il admet l'existence d'un élément démonstratif dans la perception. À moins que ce soit le noème perceptuel qui soit incompatible avec la conception que Husserl défendait dans les Recherches Logiques. Cela serait 
en accord avec l'interprétation de Dummett suivant laquelle le noème perceptuel ne tient compte que de la manière par laquelle le percevant conçoit (erfaßt) les objets extérieurs. Mais cette interprétation va à l'encontre de la $\$ 131$ des Idées Directrices où Husserl distingue clairement deux composantes du «sens noématique», à savoir le « $X$ » qui correspond à l'élément démonstratif dont nous venons de parler, et le "Wie», c'està-dire l'agrégat de descriptions qui détermine la manière par laquelle l'objet est conçu. Or ces deux composantes du noème sont irréductibles.

On peut invoquer ici l'argument de la possibilité de l'erreur qu'illustre le cas d'une perception trompeuse afin de justifier cette irréductibilité. Il concerne cette troisième propriété que Dummett attribue au noème perceptuel, à savoir que celui-ci peut changer alors que l'objet peut rester le même. Il ne s'agit pas ici de l'objet pur et simple, c'est-à-dire de quelque chose de déjà constitué, mais de "l'objet visé », du corrélat d'un acte de perception. Car la fonction du noème est de "prescrire » l'objet d'un acte - ce qui veut dire qu'au même noème doit correspondre un même objet. Or, comme le montre le cas d'une perception trompeuse, il faut composer avec la possibilité qu'une perception d'un objet soit incompatible avec d'autres perceptions visant le même objet. C'est dans ce cas précis que l'on peut dire qu'un acte peut avoir un contenu sans que lui corresponde rien de réel. La conscience se comporte alors comme si le corrélat était quelque chose de réel. Maintenant, si l'on peut dire que ce cas fournit un argument pour l'irréductibilité de la composante descriptive et de la composante indexicale, c'est que l'on peut référer à quelque chose sans que le référant satisfasse le contenu descriptif. Ce que Husserl [1950: §131] appelle le " $X$ » est ce en vertu de quoi la conscience garde le contact avec l'objet visé et ce abstraction faite de toute prédication.

Un autre aspect de la perception que Dummett examine concerne les notions d'anticipation et de remplissement, c'està-dire la composante par laquelle un acte de perception vise son objet et celle par laquelle il se remplit. La première composante est comparable à un élément hypothétique dans l'acte qui fait que la perception va toujours au-delà de ce qui est donné en elle. C'est d'ailleurs ce qui amène Dummett à comparer le noème à un "réseau d'anticipations" qui informe notre expérience du monde matériel. Ainsi, 
qu'un acte de perception doive contenir un noème, cela revient à exiger que l'on puisse toujours percevoir un objet comme quelque chose qui possède telles caractéristiques, par exemple comme quelque chose qui est d'un genre particulier, ou comme quelque chose qui a une forme tri-dimentionnelle déterminée, ou encore comme quelque chose qui tend à se comporter d'une manière déterminée. [1988: 101]

Le fait, par exemple, que l'on puisse reconnaître une ellipse sans en connaître sa caractérisation géométrique, ou encore le fait que l'on s'attende à ce qu'un objet dont la base est convexe basculera si placé sur une surface plane, semblent impliquer la présence de ce type d'anticipations. Dummett semble également impressionné par la façon spontanée avec laquelle nous interprétons nos impressions visuelles et que nous attribuons formes, couleurs, etc., aux objets. À cet égard, Dummett [1988: 102] reconnaît l'intérêt que présentent les notions de "type " et "d'horizon " qui jouent un rôle central dans la théorie de l'intentionnalité de Husserl ${ }^{6}$.

\section{V}

Si l'on admet que l'élément indexical contenu dans le noème perceptuel rend compte de la sensibilité du contenu intentionnel aux déterminations sociales et physiques par exemple, quelle solution Dummett attribue-t-il à Frege en ce qui a trait au problème de l'idéalisme? Elle consiste à attribuer au langage naturel le fait que certaines expressions ont un sens sans référence et à construire un langage parfait afin de pallier cette «carence ». Il faut donc s'assurer qu'à tout sens correspond une référence. Pour Frege, la référence d'une proposition est en ceci garantie que toute pensée, et par conséquent la proposition qui l'exprime, est vraie ou fausse en soi, c'est-à-dire indépendamment de l'aptitude d'un agent à reconnaitre que c'est le cas. Mais qu'en est-il de la référence aux objets concrets? Dans ce cas, précise Dummett [1981: 141], il n'est pas nécessaire de justifier l'usage des termes singuliers car nous possédons la «faculté de percevoir » qui nous permet d'observer les objets réels. Et c'est dans ce cas précis que s'impose le besoin d'une théorie de la perception dont on retrouve l'esquisse dans « Der Gedanke ».

Il s'agit d'un passage où Frege insiste sur le rôle du sens et de la "pensée conceptuelle" dans notre perception du monde matériel. Frege écrit:

[Les seules impressions sensibles] ne nous ouvrent pas le monde extérieur. [...] Avoir une impression sensible n'est pas encore 
voir des choses. Comment se fait-il que je vois l'arbre où je le vois? Cela tient évidemment à mes impressions sensibles et à leur nature particulière, au fait qu'elles sont produites par la vision binoculaire. Une image particulière se produit sur chacune des deux rétines - pour parler en physicien. Un autre homme voit l'arbre à la même place, il a également deux images rétiniennes, mais elles diffèrent des miennes. Nous devons admettre que ces images rétiniennes sont déterminantes pour nos impressions visuelles, mais elles diffèrent de manière notable. Et pourtant nous évoluons dans le même monde extérieur. La possession d'une impression visuelle est nécessaire à la vision des choses mais non suffisante. Ce qui doit s'y ajouter n'est pas sensible. [1970: 192]

Ce passage est plutôt elliptique mais Frege [1970: 190-1] laisse clairement entendre que la perception sensible doit contenir quelque chose de non sensible et en l'occurrence, la «saisie [Fassen] d'un sens". Ce qui veut dire que pour comprendre toute la portée de ces remarques de Frege sur la perception, il faut d'abord savoir ce qu'il entend par «saisir le sens". Tel est l'objet du chapitre 10 .

Dummett relève deux usages différents du concept " saisie du sens " par Frege. Le premier est ce qu'il appelle la "Gegebenheitsweise ", c'est-à-dire que le sens (d'une expression) est le «mode de présentation » de la référence (de cette expression). D'autre part, Frege [1970: 104] la désigne parfois par la "Bestimmtheitsweise ", c'est-à-dire la manière par laquelle la référence est déterminée. Dans ce chapitre, Dummett privilégie le second sens et il oppose deux points de vue sur la compréhension: d'une part, le réalisme de Frege suivant lequel la référence est déterminée indépendamment de nos moyens de recognition; d'autre part, le point de vue vérificationniste (brut) que fait sien Dummett et suivant lequel la référence est au contraire déterminée au moyen de nos capacités ou aptitudes (Fähigkeiten) de recognition. De là les deux sens du mot "saisir " (fassen): le premier est extensionnel, et il correspond à ce qu'il appelle une conception "épisodique » de la compréhension, conception suivant laquelle saisir le sens d'un énoncé c'est avoir le penchant de se comporter de telle manière devant une phrase donnée. Le second sens de "saisie » est intensionnelle et il est qualifié de «dispositionnel " car il repose sur «notre aptitude à comprendre une pensée (Gedanke) par la connaissance préalable du sens des parties de cette phrase ». [1988: 89] En d'autres mots, elle consiste en l'aptitude à connaître la référence d'une phrase qui contient le mot en question. Dummett rejette bien sûr la 
conception réaliste de Frege et il suggère que si ce dernier n'a pas envisagé la conception dispositionnelle de la saisie du sens, c'est qu'elle va à l'encontre de la primauté (ontologique) relative de la pensée sur le langage.

Revenons maintenant à notre question initiale: pourquoi Frege considère-t-il que la perception sensible contient la saisie d'un sens? Dummett répond que c'est vraisemblablement parce que:

la connaissance d'un ou de plusieurs objets est normalement nécessaire à la perception sensible, mais que nous ne pouvons pas «simplement percevoir» un objet en ce sens que la condition du percevoir se laisserait décrire exhaustivement par l'indication de l'objet perçu. Cet objet doit nous être donné d'une certaine manière et la manière par laquelle il nous est donné est toujours un sens qui, en retour, peut être une composante d'une pensée. [1988: 93]

Dummett semble embarrassé parce que la portée que Frege donne au concept de "sens" cadre mal avec la conception qu'il lui attribue concernant la relation entre le sens, qui, en principe, est une composante de la pensée, et le langage. Car suivant l'interprétation de Dummett [1988: 14], on ne peut saisir que les pensées qui sont conçues comme sens d'une phrase et nous n'avons accès à ces pensées que dans et par le langage. Or, si la perception sensible contient la «saisie d'un sens " comme le laisse entendre Frege, et si, comme le reconnait Dummett [1988: 103], elle engage tout l'appareil conceptuel sous-jacent à la théorie de Husserl, alors qu'advient-il de l'autonomie du langage et de la signification relativement à la pensée conceptuelle?

Face à cette question, Dummett introduit une distinction visant à préserver l'autonomie du langage et le caractère intrinsèquement linguistique du sens. Il s'agit de la distinction entre "pensée " au sens où l'entend Frege et ce qu'il appelle «les pensées primitives ( (Protogedanken) qui sont pré-expressives. "La différence essentielle repose sur le fait que ces dernières sont inséparables de l'activité et des circonstances " (Dummett [1988: 106]). Cette distinction lui permettrait donc de rendre compte des significations au sens élargi dont il a besoin pour expliquer la perception sans toutefois compromettre l'autonomie des "significations exprimables", c'est-à-dire des pensées au sens propre du terme. Et c'est précisément sur la base de cette distinction qu'il reproche à Husserl de ne pas séparer 
clairement ce qui est exprimable (le sens noématique) de ce qui ne l'est pas (les couches autour de ce noyau). Néanmoins, Dummett [1988: 109] reconnaît qu'il est possible d'attribuer au niveau pré-linguistique des Protogedanken les concepts que Husserl assigne au noème perceptuel ${ }^{7}$. Il s'agit plus particulièrement des concepts de «type» et «d'horizon» auxquels Dummett [1988: 102-3] fait allusion.

Mais cette distinction entre "Gedanke " et "Protogedanke" est-elle légitime? Cette question soulève tout le problème du rapport entre pensée et croyance. Dummett soutient évidemment que ces concepts sont essentiellement linguistiques. L'argument principal de Dummett [1988: 111, 125], peut-être le seul, repose sur la présupposition qu'à un être sans langage il manque plusieurs concepts exprimables linguistiquement. Par exemple, Dummett fait valoir qu'un animal ne pourrait pas avoir le souvenir d'un événement spécifique du passé car il ne dispose pas, en principe, des concepts qui sont nécessaires à la localisation d'un tel événement. Un animal ne peut donc pas entretenir de pensées au sens propre du terme car il ne possède que des concepts primitifs. Pour évaluer si l'argument de Dummett est légitime, il faut reprendre toute la discussion portant sur la question de savoir si les pensées et les croyances sont des concepts essentiellement linguistiques. C'est ce que nous examinerons à la section suivante.

\section{VI}

Dans son article «Philosophy of thought and philosophy of language», Dummett [1986: 153] attribue à G. Evans le mérite d'avoir perçu que la théorie frégéenne du sens présuppose un niveau sous-jacent de pensées non linguistiques, ce qu'il appelle ici les "Protogedanken", à l'aide duquel il cherche à expliquer la signification. Comme nous l'avons déjà dit, l'une des raisons de parler de pensées pré-linguistiques tient à la "qualité cognitive" de l'expérience perceptuelle, c'est-à-dire ce qui fait de celle-ci notre principal «canal d'information». L'examen de la position de Evans, qui est en fait le représentant d'une tendance qui va en s'accentuant dans la philosophie contemporaine, fait l'objet du chapitre intitulé "Pensée et langage». Ce qui fait de Evans un philosophe de la pensée, c'est précisément la tentative

d'analyser différentes manières de penser un objet pour ensuite entreprendre d'expliquer, à l'aide de ces différentes manières 
de penser un objet, les différents véhicules [Mittel] linguistiques de la référence à un objet. [1988: 11]

Evans s'intéresse donc au premier chef à l'"aboutness ", c'està-dire à ce que c'est "to think about something". En ce sens, son entreprise se rapproche singulièrement de celle de Husserl et l'on peut s'attendre à ce que les mêmes objections qui ont été soulevées contre la phénoménologie s'appliqueront également à la position que défend Evans.

Son reproche principal nous reconduit à la question du psychologisme. En effet, le danger qui guette ce type d'entreprise est de rechuter dans une forme de psychologisme du type de celle dénoncée par Frege. Selon Frege [1962: XV], elle consiste, d'une part, à relier les lois de la logique au processus de la pensée qui obéit aux lois de l'association; elle consiste, d'autre part, à réduire la pensée et le sens à de simples représentations. Car en recourant au niveau pré-linguistique de la pensée, et en cherchant à expliquer la signification par référence à la manière dont les contenus de pensée sont exprimés, on court toujours le risque, inévitable selon Dummett, de reculer jusqu'aux processus de conscience.

Ce qui est intéressant ici, c'est que la conception du langage à laquelle semblent s'engager tous ceux qui recourent au psychologique afin d'expliquer le langage est celle qui lui assigne la fonction de servir des fins de communication. $\grave{A}$ ce titre, le langage serait un code ${ }^{8}$. Les deux principales objections qui sont dirigées contre cette conception du langage comme code portent sur l'objectivité de la signification et plus généralement du contenu de pensée et sur l'objectivité de l'entente (Verständigung). Dans le premier cas, Dummett [1988: 19] fait valoir la thèse de la non-appartenance des contenus de pensée à la conscience dont il a été question plus haut. Un contenu de pensée est quelque chose d'essentiellement objectif. Cependant, comme le remarque justement Dummett, garantir l'objectivité du sens et de la pensée en faisant appel à la mythologie platonicienne comme le fait Frege est une chose, rendre compte de l'objectivité de l'entente en est une autre. En effet, il faut également être en mesure d'expliquer que, au sein d'une communauté linguistique donnée, tel sens est normalement associé à telle expérience. Afin d'éviter le réalisme auquel Frege souscrit, le langage doit être conçu comme le bien commun à l'usage des membres d'une communauté. Et c'est pour ne pas avoir reconnu le 
caractère public et la nature sociale du langage que Frege a conçu le langage comme un "idiolecte".

Le même reproche est adressé à Quine et à Davidson. Cela peut surprendre dans la mesure où, comme on le sait, la traduction radicale par laquelle Quine introduit sa doctrine de l'indétermination de la traduction dans Word and Object vise à réfuter la conception traditionnelle de la signification qui prétend expliquer le langage à l'aide d'entités indépendantes du langage telles la pensée, les idées innées, etc. Or, l'un des arguments de Quine contre cette conception mentaliste de la connaissance repose essentiellement sur la nature sociale et le caractère public du langage: il $n^{\prime} y$ a rien dans la signification qui transcende ce qui est observable dans le comportement verbal d'un locuteur dans des circonstances publiquement observables. Et comme le rappelle Quine [1987: 5] dans un article récent, l'indétermination de la signification est une conséquence de son seul behaviorisme, c'est-à-dire de la psychologie des stimuliréponses appliquée au langage. Aussi les seules indices dont nous disposons dans l'apprentissage d'une langue et dans la traduction radicale sont ceux que nous livre l'observation du comportement verbal et non verbal de l'agent sous observation. Le behaviorisme ainsi conçu est une position épistémologique dans la mesure où il ne concerne que l'évidence empirique et l'observation du comportement. Et il n'y a rien dans ce que nous livre le comportement de notre agent qui puisse déterminer le choix d'un manuel de traduction plutôt qu'un autre. Car deux manuels peuvent être également compatibles avec les données que nous livre l'observation du comportement verbal tout en étant radicalement différents. On pourrait interpréter la doctrine quinienne de l'indétermination de la traduction en termes d'inextricabilité de la signification et de la croyance? Ainsi, l'interprétation du comportement verbal d'un agent est relative aux croyances qui lui sont attribuées car si l'interprète lui attribue telle croyance, son comportement sera compris d'une manière très différente s'il lui attribue des états intentionnels différents. Mais cette relativité entre la signification et la croyance ne fait pas du langage un idiolecte, car l'exigence d'interprétabilité est initialement inscrite dans le contenu public et la nature sociale du langage et elle montre que, pour être compris, un locuteur doit tenir compte de l'usage qu'en fait la communauté linguistique à laquelle il appartient. 
Même en admettant cela, Dummett ne peut se satisfaire $d^{\prime}$ une doctrine pour laquelle

notre compréhension des autres dépendra d'hypothèses incontrôlables (unüberprüfbaren) sur les théories de la signification qui règlent implicitement les assertions des locuteurs. [1988: 142]

C'est précisément ce qu'il [1986: 165] reproche à la théorie véri-conditionnelle de Davidson, à savoir de ne pas être autre chose qu'une simple interprétation, qu'une "piece of theoretical knowledge » qui est irréconciliable avec la "compréhension implicite " que nous avons de notre langue. À cet égard, on peut mentionner la distinction dont se sert Dummett [1974: 100] entre une théorie "modeste " et une théorie «intégrale " (full blooded) de la signification afin de mieux comprendre le sens de sa critique. La première donne une interprétation du langage en présupposant chez l'agent la possession des concepts nécessaires à la compréhension d'une langue. Elle ne dit supposément rien sur la manière par laquelle un agent qui possède déjà une langue peut en comprendre une autre. Par contre, une théorie plus ambitieuse de la signification doit pouvoir rendre compte des aspects de la pratique linguistique qui ont trait à la connaissance implicite et à la compréhension de notre langue maternelle. Ainsi, la tâche que Dummett assigne à une théorie «intégrale» de la signification est de rendre compte de la connaissance que doit posséder un locuteur pour comprendre la signification d'une expression. Et puisque la signification est ce que l'on doit connaître afin de comprendre une expression, une théorie de la signification doit se développer parallèlement à une théorie de la compréhension [1988: 152]. Mais sur la question de la forme que devraient prendre ces deux théories, Dummett [1988: 163] ne semble pas en mesure de fournir une réponse décisive. Tout ce qu'on sait, c'est que la compréhension $\mathrm{n}^{\prime}$ est pas une simple affaire d'interprétation ${ }^{10}$.

On le voit, deux stratégies s'opposent: celle de l'interprétation et de la traduction radicale d'une part, celle, d'autre part, d'une théorie du sens dont la tâche consiste à expliciter cette connaissance qui est implicite à la compréhension d'une langue. Quine soutient au contraire que la compréhension n'est pas à expliquer en termes de connaissances linguistiques mais en termes d'observation. Or, l'observation, comme l'a reconnu Dummett, est sur-déterminée par les croyances et les anticipations. Dès lors, l'une des tâches de l'interprétation radicale consiste 
à expliciter ce qu'on appelle dans la tradition herméneutique inspirée de Husserl "l'horizon de la compréhension".

Une autre façon d'aborder ce problème est de se demander quelle est la part qui revient à la compréhension privée lorsque le langage est conçu comme une institution sociale et publique? Certes, il n'y a pas de réponse simple à cette question. Dummett [1988: 132] reconnaît qu'il faut tenir compte de la compréhension individuelle. Cependant, il soutient que la signification dépend d'abord et avant tout du bon usage et marginalement des intentions de l'agent. C'est le principe de la «division linguistique du travail " qui stipule que l'on ne peut mettre sur un même pied la compréhension privée et la signification dans la communauté linguistique. Ce qui veut dire que l'on ne peut déterminer les intentions $d^{\prime}$ un agent que sur la base de sa connaissance linguistique et du degré de compétence qu'on lui attribue. Car "c'est surtout parce que le locuteur connaît la langue [...] que nous pouvons lui attribuer linguistiquement motifs et intention.» (Dummett [1988: 151]). Il serait donc absurde d'attribuer pensées et croyances à des êtres comme des animaux par exemple qui ne disposent pas des moyens pour la manifester. Tel est, on s'en souvient, l'argument de Dummett pour la distinction entre "pensée " et " pensée primitive ». Pourtant, nous le faisons couramment et si l'on se fie à Quine [1960: 219] cela relève de notre "virtuosité dramatique». De là la stratégie de l'interprétation radicale. De ce point de vue, la question n'est pas de savoir si les animaux, par exemple, ont des croyances même s'ils n'ont pas les moyens linguistiques de les manifester; car avoir une croyance, du point de vue de l'interprète radical, c'est d'abord pouvoir être l'objet d'une attribution de croyance - attribution qui se règle sur les données que lui livre l'observation du comportement de l'agent. L'indétermination qui résulte de la relativité entre la signification et l'attribution de croyances n'est pas si dramatique dans la mesure où l'on peut lui imposer des contraintes comme l'a montré Davidson. Ce qu'il faut retenir ici, c'est que la relativité entre les concepts de signification et de croyance ne signifie en rien une rechute dans le psychologisme comme le suggère Dummett. Il ne s'agit pas ici de faire appel à l'intentionnel et aux croyances afin de déterminer la signification. Au contraire, il s'agit de se servir de la doctrine de Quine afin de justifier une attitude non réaliste et non mentaliste à l'égard des croyances. Cependant, ces croyances ne sont compatibles avec l'indétermination que si elles ne sont pas essentiellement linguistiques. 


\section{VII}

Toute la discussion du chapitre précédent autour de la relation entre pensée et langage nous ramène au principe de l'autonomie de la signification autour duquel sont organisés les arguments de Dummett relativement au primat de la philosophie du langage sur la philosophie de la pensée. Qu'en est-il de la paternité de Frege une fois que l'on admet, comme le fait Dummett [1986: 154], que la théorie frégéenne de la signification présuppose un niveau de pensées pré-linguistiques qui s'est avéré nécessaire afin de rendre compte de l'expérience perceptuelle? Cette théorie ne peut constituer la thèse fondamentale de la philosophie du langage car elle compromet l'autonomie de la signification. Car si l'on admet avec Frege la priorité de la pensée sur le langage, il faut présupposer que la saisie du sens précède l'acquisition du langage. Et si la saisie du sens précède l'acquisition du langage, il est tentant de faire valoir contre Frege les objections dont Dummett s'est servi afin de réduire la phénoménologie de Husserl à une forme de psychologisme. $C^{\prime}$ est ce que fait $H$. Putnam [1975: 219] qui résume la conception frégéenne de la signification en disant que la signification est une entité idéalisée, qu'elle est saisie par un "acte psychologique " en ce sens que connaître la signification d'un terme est « just a matter of being in a psychological state». Une manière d'échapper aux objections de Putnam serait de subordonner le concept frégéen de sens à une théorie de l'intentionnalité qui ne serait pas psychologique au sens où l'entend Putnam, c'est-à-dire en ce sens qu'un état psychologique "étroit" présupposerait le principe du solipsisme méthodologique. La théorie husserlienne de l'intentionnalité est un pas dans cette direction.

En admettant cela et en reconnaissant le bien-fondé des arguments que nous avons fait valoir contre les objections de Dummett concernant l'incompatibilité de la doctrine frégéenne du sens et de la théorie husserlienne de l'intentionnalité, qu'en est-il de l'argument de Dummett pour la distinction entre "Gedanke " et "Protogedanke "? Un organisme sans langage qui ne disposerait que de "concepts primitifs" pourrait-il avoir des pensées au sens propre du terme? Un organisme peut-il entretenir de telles pensées s'il n'a pas les moyens linguistiques de les manifester? En d'autres mots, les pensées ont-elles un caractère essentiellement linguistique? Cette question que nous avons à peine effleurée au chapitre précédent serait à reprendre 
dans le contexte de la relation entre pensée et croyance. Et comme je l'ai suggéré, j'estime que cette question ne peut être tranchée en faisant appel à une théorie de la signification d'un langage.

Département de philosophie

Université du Québec à Montréal

\section{NOTES}

1. La version anglaise de ce texte est parue sous le titre "The origins of analytical philosophy " (1) et (2) dans la revue Lingua e Stile 23, $\mathrm{n}^{\circ} \mathrm{s} 1$ et 2, 1988, pp. 3-49 et pp. 171-210.

2. M. Dummett, Frege: Philosophy of Language, Cambridge, Harvard University Press, 1973 et The Interpretation of Frege's Philosophy. Cambridge, Harvard University Press, 1981.

3. J'ai discuté de cette question dans mon article « Husserl et Frege: Les remarques de M. Dummet sur la généralisation de la signification».

4. Sur les arguments justifiant le passage d'une conception de la signification comme "essence d'acte » à la conception noématique de la signification, voir E. Husserl [1987] et D. Fisette "Signification et essence: les Leçons de 1908 de E. Husserl sur sa doctrine de la signification ", Dialogue, XXX, 1991, pp. 33-49.

5. La conception Humpty-Dumpty de la signification se résume dans cette phrase de L. Carroll: « Lorsque j'emploie un mot, il signifie exactement ce que je veux - ni plus, ni moins », in Dummett [1988: 45]. Voir également Dummett [1988: 85] pour le rapprochement de cette conception avec la doctrine des Recherches logiques.

6. Sur les concepts de "type " et d' " horizon ", voir entre autres E. Husserl [1947: 32-47], [1970 35-45, 146-149, 401-406].

7. Je rappelle toutefois que le niveau pré-linguistique des pensées primitives que Dummett est disposé à reconnaître est de type essentiellement spatial [1988: 107-8]. Il reconnaît par exemple que la forme tridimensionnelle de l'objet est indissociable de la perception. Cette caractéristique et d'autres (la cohésion de l'objet par exemple) ont quelque chose à voir avec les anticipations qui sont déclenchées dans un acte de perception.

8. Un des défenseurs de cette conception est nul autre que $F$. de Saussure qui, dans son Cours de Linguistique Générale, écrit: "Voici donc deux personnes, $A$ et $B$, qui s'entretiennent. Le point de départ du circuit est dans le cerveau de l'un, par exemple $A$, où les faits de conscience, que nous appellerons concepts, se trouvent associés aux représentations des signes linguistiques ou images 
acoustiques servant à leur expression. Supposons qu'un concept donné déclenche dans le cerveau une image acoustique correspondante; c'est un phénomène entièrement psychique, suivi à son tour d'un procès physiologique: le cerveau transmet aux organes de la phonation une impulsion corrélative à l'image; puis les ondes sonores se propagent de la bouche de $\mathrm{A}$ à l'oreille de $\mathrm{B}$ : procès purement physique. Ensuite, le circuit se prolonge en B dans un ordre inverse: de l'oreille au cerveau, transmission physiologique de l'image acoustique; dans le cerveau, association psychique de cette image avec le concept correspondant ", cité par Dummett [1988: 117]. Dans un commentaire sur M. Dummett [1986], P. Engel [1986: 157] remarque justement que J. Fodor tombe également sous cette catégorie.

9. C'est d'ailleurs Dummett qui le premier a parlé de la doctrine quinienne de l'indétermination de la traduction en termes d' " inextricabilité ", c'est-à-dire la thèse suivant laquelle il n'y a pas une connaissance de la signification qui ne serait pas contaminée par les croyances. Sur les arguments de Dummett contre la thèse de l'inextricabilité, voir son article "The significance of Quine's indeterminacy Thesis ", dans M. Dummett [1978].

10. Sur cette question, je me permets de renvoyer à D. Laurier [1987] qui semble aller dans le sens de Davidson en suggérant l'idée d'une théorie "simultanée " de la signification et de la croyance (pp. 31-32). Sur la discussion concernant l'opposition entre le molécularisme de Dummett et le holisme, cf. J. Couture [1990].

\section{BIBLIOGRAPHIE}

COUTURE, J., "Le Molécularisme: logique et sémantique », Cahier d'épistémologie, n 9009 du Département de philosophie de l'UQAM, 1990.

DUMMETT, M., "The origins of analytical philosophy " (1) et (2) Lingua et Stile, 23, $\mathrm{n}^{\circ} \mathrm{s} 1$ et 2, pp. 3-49 et pp. 171-210, 1988.

DUMMETT, M., Ursprünge der analytischen Philosophie, Suhrkamp Verlag, Frankfurt am Main, 1988.

DUMMETT, M., "What is a theory of meaning " in S. Guttenplan (ed.) Mind and Language, London, Clarendon Press, 1975.

DUMMETT, M., "The philosophy of thought and the philosophy of language " in Mérites et Limites des Méthodes Logiques en Philosophie, Paris, Vrin, 1986.

DUMMETT, M., The Interpretation of Frege's Philosophy, Cambridge, Mass., Harvard University Press, 1981.

DUMMETT, M., Frege: Philosophy of Language, London, G. Duckworth, 1973. 
DUMMETT, M., Truth and other Enigmas, Cambridge, Harvard University Press, 1978.

ENGEL, P., «Rapport» sur "The philosophy of thought and the philosophy of language » de M. Dummett in Mérites et Limites des Méthodes Logiques en Philosophie, Paris, Vrin, pp. 155-165. 1986.

FREGE, G., Écrits Logiques et Philosophiques, Paris, Seuil, 1970.

FREGE, G., Grundgesetze der Arithmetik, Hildesheim, G. Olms, 1962.

HUSSERL, E, Expérience et Jugement: recherches en vue d'une généalogie de la logique, Paris, Presses Universitaires de France, 1970.

HUSSERL, E., Vorlesungen über Bedeutungslehre: Sommersemester 1908, Dordrecht, M. Nijhoff, 1987.

HUSSERL, E., Idées Directrices pour une Phénoménologie et une Philosophie Phénoménologique Pure, Paris, Gallimard, 1950.

HUSSERL, E., Recherches Logiques, Paris, Presses Universitaires de France, 1969.

HUSSERL, E., Méditations Cartésiennes: Introduction à la Phénoménologie, Paris, Vrin, 1947.

LAURIER, D., “Comprendre ou interpréter», Cahiers n $8906 \mathrm{du}$ Département de Philosophie de l'Université de Montréal, 1989.

PUTNAM, H., "The Meaning of "Meaning ", Philosophical Papers, vol. II, Cambridge, Cambridge University Press, 1975.

QUINE, W., Word and Object, Cambridge, Mass., MIT Press, 1960.

QUINE, W., "Indeterminacy of translation again ", Journal of Philosophy, LXXXIV, 1987. 